Check for updates

Cite this: J. Mater. Chem. A, 2021, 9 , 24283

Received 17th July 2021

Accepted 22nd October 2021

DOI: $10.1039 / \mathrm{d} 1 \mathrm{ta0} 0032 \mathrm{e}$

rsc.li/materials-a

\section{Rapid synthesis of supported single metal nanoparticles and effective removal of stabilizing ligands $\uparrow$}

\author{
Panagiotis Trogadas, (D) $\ddagger^{\star a}$ Nidhi Kapil, (D) $\ddagger^{a}$ Gyen Ming A. Angel, (D) ${ }^{b}$ Stefanie Kühl, $\S^{c}$ \\ Peter Strasser, (iD ${ }^{c}$ Dan J. L. Brett (iD ${ }^{b}$ and Marc-Olivier Coppens (D) $^{a}$
}

\begin{abstract}
A method is introduced to rapidly ( $<30 \mathrm{~min}$ ) synthesize single metal nanoparticles with narrow size distribution in a simple way. It is based on the electrospraying of a metal precursor solution into a surfactant solution, which acts as a reducing and stabilizing agent. This synthesis method is demonstrated for the production of $\mathrm{Ag}$ and $\mathrm{Au}$ nanoparticles, which are incorporated onto carbonaceous and noncarbonaceous supports. The nanoparticle size depends on the internal diameter of the spraying nozzle. The removal of the stabilizing surfactant (dodecylamine; DDA) is also examined via thermal annealing and oxygen plasma treatments. Thermal annealing at a low temperature rate is found to be the most effective, as it completely removes DDA from the metal nanoparticles without inducing changes in their particle size. To verify that the supported Ag nanoparticles post calcination are surfactant-free and, thus, their surface sites are active, their oxygen reduction reaction (ORR) activity is measured in alkaline media, demonstrating similar values to the ones reported in the literature.
\end{abstract}

\section{Introduction}

Metal nanoparticles currently receive significant attention due to their prominent properties, dictated by their size, shape, and crystalline structure, which are attractive to various technological applications, such as catalysis, ${ }^{1-3}$ healthcare, ${ }^{4}$ sensing, ${ }^{5}$ and energy ${ }^{6-8}$ Diverse methods are employed for the synthesis of

${ }^{a}$ EPSRC "Frontier Engineering" Centre for Nature Inspired Engineering, Department of Chemical Engineering, University College London, London, UK. E-mail: p.trogadas@ ucl.ac.uk

${ }^{b}$ Electrochemical Innovation Lab, Department of Chemical Engineering, University College London, London, UK

${ }^{'}$ The Electrochemical Energy, Catalysis and Materials Science Laboratory, Department of Chemistry, Chemical Engineering Division, Technical University Berlin, Germany $\dagger$ Electronic supplementary information (ESI) available. See DOI: 10.1039/d1ta06032e

\$ Both authors contributed equally.

$\S$ Present address: Department of Interface Science, Fritz-Haber-Institute of the Max-Planck Society, Berlin, Germany. metal nanoparticles, categorized into top-down and bottom-up approaches. ${ }^{4,8}$ Their main difference is the starting material for the formation of nanoparticles. In top-down approaches, bulk material is reduced to nanoparticles via physical, chemical or mechanical processes, such as milling, ion sputtering and chemical etching, whereas, in the case of bottom-up approaches (vapor deposition, sol-gel, pyrolysis, aerosol, etc.), atoms or molecules are the starting point for the creation of nanoparticles, in which building blocks of nanoparticles are initially formed and then assembled to produce the final form of nanoparticle., ${ }^{4,8}$

Among these synthesis methods, the colloidal approach is one of the most widely used for the development of heterogeneous catalysts. ${ }^{9}$ It allows accurate control of the particle size, shape, and structure using surfactants as capping and stabilizing agents. Even though these ligands are indispensable in the creation of nanoparticles, as they stabilize the high-energy surface of the nanoparticles prohibiting aggregation, ${ }^{10-12}$ they significantly decrease their activity, since they block the access of reactants to active sites. ${ }^{13}$ Solvent extraction and thermal decomposition are usually employed for the removal of these ligands, resulting in the agglomeration of the ligand-free nanoparticles., ${ }^{9,13}$

However, the precise synthesis of single metal nanoparticles based on bottom-up and top-down approaches is challenging, as it involves multiple reagents and interdependent experimental conditions, such as reagent concentration, reaction time, temperature and mixing efficiency. ${ }^{14}$ The effect of each experimental variable on the properties of the nanoparticles is determined by trial and error, so that the identification of optimal reaction conditions becomes tedious, time consuming, and resource intensive. Its sensitivity towards variations in reaction conditions and the presence of impurities in the solution result in reproducibility issues. ${ }^{14,15}$ Hence, more controlled approaches have been introduced for the synthesis of nanoparticles with specific characteristics, relying on artificial intelligence, ${ }^{14}$ bio-imitation, ${ }^{16}$ and electrospraying. ${ }^{17}$

In this work, we present an underestimated technique, electrospraying (Table 1), as an alternative method to the 
Table 1 Comparison of different methods for the synthesis of metal nanoparticles

\begin{tabular}{|c|c|c|}
\hline Synthesis of metal nanoparticles & Advantages & Disadvantages \\
\hline Top-down methods & $\begin{array}{l}\text { - Simple and efficient } \\
\text { - Scalable } \\
\text { - Chemical purification not required }\end{array}$ & $\begin{array}{l}\text { - Alteration of surface chemistry and properties } \\
\text { of nanoparticles } \\
\text { - Time-consuming } \\
\text { - Contamination risk } \\
\text { - Cost }\end{array}$ \\
\hline Bottom-up methods & $\begin{array}{l}\text { - Accurate control of shape and size } \\
\text { - Nanoparticles have a structure with less } \\
\text { defects, and better short- and long-range } \\
\text { ordering than nanoparticles produced by top- } \\
\text { down methods } \\
\text { - Cost-efficient }\end{array}$ & $\begin{array}{l}\text { - Limited scalability } \\
\text { - Chemical purification required }\end{array}$ \\
\hline Proposed method & $\begin{array}{l}\text { - Accurate control of shape and size } \\
\text { - Rapid } \\
\text { - Operation at room temperature } \\
\text { - Presence of only two reactants }\end{array}$ & $\begin{array}{l}\text { - Limited scalability } \\
\text { - Chemical purification required }\end{array}$ \\
\hline
\end{tabular}

colloidal approach for the facile synthesis $(<30 \mathrm{~min})$ of metal nanoparticles with controlled size involving the use of surfactants as capping agents. It is based on spraying, at a high applied voltage (greater than $9 \mathrm{kV}$ ), a metallic precursor solution into a liquid solution containing surfactants, which act as reducing and stabilizing agents at the same time. The advantages of this method include the presence of only two reactants (i.e., metal precursor and reductive/stabilizing agent) for the synthesis of metal nanoparticles, rapid reaction between the precursor and the reductive solution, and operation at room temperature. However, scale-up remains a challenge, requiring complex multi-nozzle systems. Herein, we utilize this synthesis method to produce single $\mathrm{Au}$ and $\mathrm{Ag}$ nanoparticles, which are then incorporated onto supports. Dodecylamine (DDA) is used as the reducing and stabilizing agent. Its efficient removal from the supported metal nanoparticles, without altering their particle size, is investigated. The effect of the spraying nozzle diameter on the size of the nanoparticles produced is also examined.

\section{Controlled synthesis of metal nanoparticles}

The metal precursor solution is prepared by dissolving gold chloride trihydrate $\left(13 \mathrm{mg}, \mathrm{HAuCl}_{4} \cdot 3 \mathrm{H}_{2} \mathrm{O}\right)$ or silver nitrate $\left(\mathrm{AgNO}_{3}\right)$ in isopropanol $(15 \mathrm{~mL})$. DI water cannot be used as a solvent, due to its high surface tension and electrical conductivity, impeding stable electrospraying. ${ }^{18}$ The reductive surfactant solution is prepared in two steps: (1) dodecylamine $(1.5 \mathrm{~g})$ is dissolved in cyclohexane $(50 \mathrm{~mL})$ after vigorous stirring for $10 \mathrm{~min}$ at room temperature, and then (2) formaldehyde (12 $\mathrm{mL}$ ) is added to the above solution. The mixture is continuously stirred for another $10 \mathrm{~min}$ at room temperature, resulting in the formation of dodecylaminomethanol (DDAM; eqn (1)) and washed twice with DI water to remove the cyclohexane:

$$
\begin{aligned}
\mathrm{CH}_{3}\left(\mathrm{CH}_{2}\right)_{10} \mathrm{CH}_{2} \mathrm{NH}_{2}+\mathrm{HCHO} \rightarrow & \rightarrow \mathrm{CH}_{3}\left(\mathrm{CH}_{2}\right)_{10} \mathrm{CH}_{2} \mathrm{NHCH}_{2} \mathrm{OH}
\end{aligned}
$$

For each electrospraying experiment (Fig. S1†), a glass syringe $(10 \mathrm{~mL})$ is filled with metal precursor solution $(5 \mathrm{~mL})$ and placed on a syringe pump, which injects the precursor solution at a constant flow rate $\left(0.5 \mathrm{~mL} \mathrm{~h}^{-1}\right)$ into the DDAM solution $(20 \mathrm{~mL})$ via an electrified stainless-steel nozzle with a flat rim. A chemically resistant tube connects the syringe with the stainless-steel nozzle. Four nozzles with different internal diameters (i.e., $0.007,0.020,0.030$, and 0.046 inches) are used to evaluate their effect on the size of the produced metal nanoparticles. The applied voltage between the stainless-steel nozzle and counter electrode ring (soldering alloy-Sn : $\mathrm{Cu} 99.3: 0.7$ ) positioned into the DDAM solution is $9.5 \mathrm{kV}$ to generate the precursor aerosol. The DDAM solution is placed at least $5 \mathrm{~cm}$ away from the electrified stainless-steel nozzle (optimal distance) to avoid the creation of electric sparks and ensure the stability of the electrospraying jet. The electrospraying is performed for 30 minutes under constant stirring and the solution turns pink or yellow (Fig. S2 $\dagger$ ), indicating the formation of gold or silver nanoparticles, respectively.

Once the metal precursor solution comes in contact with the reductive surfactant solution, the $\mathrm{NHCH}_{2} \mathrm{OH}$ group of DDAM (eqn (1)) is oxidized to unstable NHCOOH. As a result, DDA is released and adsorbed on the surface of metal ( $\mathrm{Ag}$ or $\mathrm{Au})$ nanoparticles (Fig. S16†), serving as a protecting agent (eqn (2) and (3)):19,20

$$
\begin{array}{r}
\mathrm{CH}_{3}\left(\mathrm{CH}_{2}\right)_{10} \mathrm{CH}_{2} \mathrm{NHCH}_{2} \mathrm{OH}+\mathrm{HAuCl}_{4} \cdot 3 \mathrm{H}_{2} \mathrm{O} \rightarrow \\
\mathrm{CH}_{3}\left(\mathrm{CH}_{2}\right)_{10} \mathrm{CH}_{2} \mathrm{NH}_{2}+\mathrm{Au}+\mathrm{HCOOH} \\
\mathrm{CH}_{3}\left(\mathrm{CH}_{2}\right)_{10} \mathrm{CH}_{2} \mathrm{NHCH}_{2} \mathrm{OH}+\mathrm{AgNO}_{3} \rightarrow \\
\mathrm{CH}_{3}\left(\mathrm{CH}_{2}\right)_{10} \mathrm{CH}_{2} \mathrm{NH}_{2}+\mathrm{Ag}+\mathrm{HCOOH}
\end{array}
$$

\section{Results and discussion}

The presence of metal nanoparticles in the cyclohexane solution is confirmed via UV-vis spectroscopy, revealing the existence of the characteristic absorption peaks of $\mathrm{Ag}$ and $\mathrm{Au}$ nanoparticles at 402 and $520 \mathrm{~nm}$, respectively (Fig. 1(a) and (b))..$^{19,21-23}$ As the 

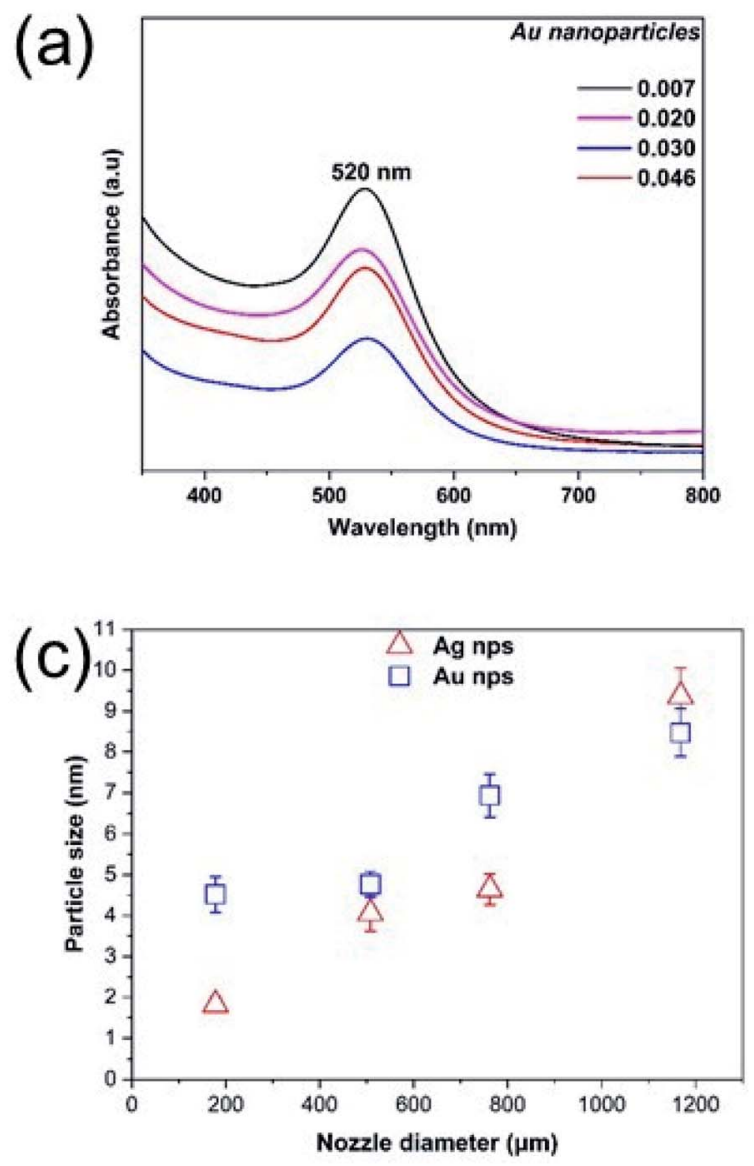
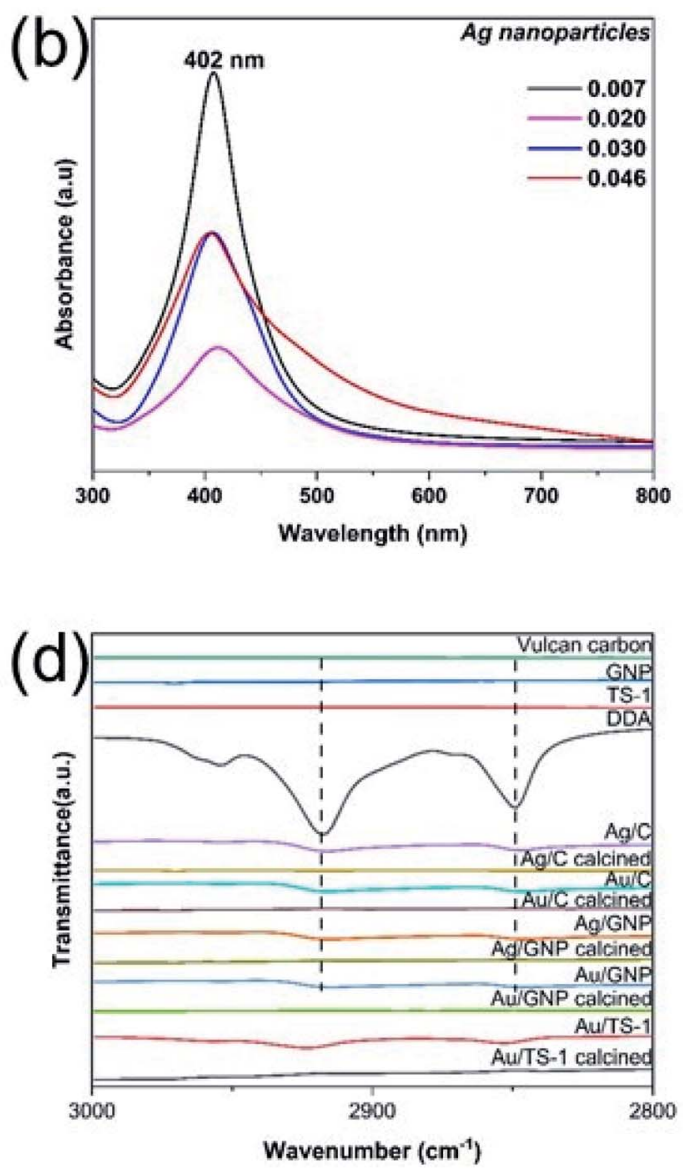

Fig. 1 (a and b) UV-vis spectra of synthesized Au and Ag nanoparticles, using different nozzles via electrospraying. (c) The particle size of Ag and Au nanoparticles is directly affected by the internal diameter of the spraying nozzle. (d) The adsorption of DDA surfactant onto the as-synthesized supported metal nanoparticles is evident in FTIR spectra.

diameter of the electrospraying nozzle is increased above 0.030 inches, there is a small shift of the absorption peak towards a higher wavelength, indicating the formation of larger nanoparticles. ${ }^{24}$ As the particle size increases, the surface plasmon resonance peak heights gradually decrease and peak widths broaden. ${ }^{25}$ High-resolution TEM measurements demonstrate the formation of quasi-spherical $\mathrm{Ag}$ and $\mathrm{Au}$ nanoparticles with $\{222\}$ and $\{200\}$ facets, ${ }^{26,27}$ respectively (Fig. S3 and S4 $\dagger$ ).

The produced $\mathrm{Ag}$ and $\mathrm{Au}$ nanoparticles (Table $\mathrm{S} 1 \dagger$ ) are monodisperse and have narrow distributions centering at $1.8 \pm$ $0.2 \mathrm{~nm}$ and $4.5 \pm 0.4 \mathrm{~nm}$, respectively, for the smallest internal diameter of electrospraying nozzle (0.007 inch) and $9.4 \pm 0.7$ and $8.5 \pm 0.6 \mathrm{~nm}$, respectively, for the largest internal diameter of electrospraying nozzle (0.046 inch). The average distance between neighboring nanoparticles is approximately $2 \mathrm{~nm}$, double the length of fully extended dodecylamine. ${ }^{19}$ No alteration in their particle size is observed after storing these metal nanoparticle solutions for eight months (Fig. S5 $\dagger$ ), demonstrating their good stability. If dripping is used instead (i.e., $0 \mathrm{~V}$ applied voltage), agglomerated large clusters of metal nanoparticles are formed (Fig. S6†).

It is evident that the size of the synthesized nanoparticles is directly dependent on the diameter of the employed electrospraying nozzle (Fig. 1(c) and S15 $\dagger$ ). As the diameter of the base of the formed Taylor cone corresponds to the internal diameter of the electrospraying nozzle (Fig. S15 $\dagger$ ), the jet injected out of the nozzle with the smallest internal diameter (i.e. 0.007 inch) is finer than the jets produced from the other nozzles, resulting in the synthesis of nanoparticles with a narrow size distribution (Fig. $\mathrm{S} 7$ and $\mathrm{S} 8 \dagger$ ). ${ }^{28-30}$ As a result, $\mathrm{Ag}$ and $\mathrm{Au}$ nanoparticles produced via electrospraying with the 0.007 inch nozzle have the smallest particle size. This observation is in accordance with previous results reported in the literature, ${ }^{28-30}$ where electrospraying nozzles with different internal diameter are used for the formation of monodisperse polymer beads.

The synthesized metal nanoparticles are then supported on carbonaceous and non-carbonaceous supports, namely Vulcan carbon, graphene nanoplatelets, and TS-1 (Fig. S9†), via the addition of the chosen support $(10 \mathrm{mg})$ into the metal nanoparticle solution $(2 \mathrm{~mL})$ and vigorous stirring for $2 \mathrm{~h}$ at room temperature. The liquid solution is then evaporated using a rotary evaporator and the samples are dried at room temperature overnight (ESI Section S1.3†). FTIR spectra of these untreated supported metal nanoparticles present two peaks at $\sim 2930 \mathrm{~cm}^{-1}$ and $2858 \mathrm{~cm}^{-1}$, similar to the ones in the spectrum 
of pristine DDA (Fig. 1(d)), due to the adsorption of DDA onto the supports, resulting in stretching vibration of alkyl chains $\mathrm{C}-$ H. ${ }^{31}$

Despite the stabilizing role of DDA, it is essential to successfully remove it from the supported metal nanoparticles without altering their particle size, as it blocks access to their active sites, rendering them inactive in catalytic applications. ${ }^{11,20}$ Thus, thermogravimetric analysis (TGA) is employed to calculate the heating temperature necessary for the removal of surfactant. A weight loss of up to $40 \%$ occurs during the isothermal process at $350{ }^{\circ} \mathrm{C}$ for the supported metal nanoparticles, due to the removal of organic surfactants (Fig. 2(a) and (b)). At higher temperatures, there are no additional organic substances present in the samples, as no substantial loss in weight is observed with further increase of the temperature up to $700{ }^{\circ} \mathrm{C}$. Thus, thermal annealing at $350{ }^{\circ} \mathrm{C}$ in air is efficient for removing organic surfactants from the surface of the metal nanoparticles.

TGA measurements (Fig. 2, S10 and S11†) showcase that a significant weight loss occurs at higher temperature $\left(350{ }^{\circ} \mathrm{C}\right)$ than required for pure DDA; its steep slope indicates that free DDA is completely degraded at $150{ }^{\circ} \mathrm{C}$ (Fig. 2(a) and (b)). ${ }^{32,33}$ This shift in temperature indicates the multilayer adsorption of DDA on the surface of supported metal nanoparticles and the binding of amine groups to the metal nanoparticles, ${ }^{34}$ requiring a higher temperature for their removal. Vulcan carbon, graphene nanoplatelets, and TS- 1 are stable during the surfactant removal process, since no change in weight is observed for these supports, up to $350{ }^{\circ} \mathrm{C}$.

To verify the complete removal of DDA surfactant from supported metal nanoparticles post calcination, various characterization methods are utilized (ESI Section S1.4†), including differential thermal analysis (TG-DTA), Fourier transform infrared spectroscopy (FTIR), and X-ray photoelectron spectroscopy (XPS).

DTA analysis demonstrates the absence of characteristic DDA peak appearing between $100{ }^{\circ} \mathrm{C}$ and $200{ }^{\circ} \mathrm{C}$ and chemisorbed DDA on supports between $300{ }^{\circ} \mathrm{C}$ and $350{ }^{\circ} \mathrm{C}$ from all calcined supported metal nanoparticles (Fig. 2(c), (d), S10(c), (d) and $\mathrm{S} 11 \dagger)$. Furthermore, the absence of DDA peaks from FTIR (Fig. 1(d)) and XPS (Fig. S13†) C 1s spectra (C-N peak at $\sim 285.5 \mathrm{eV}$ (ref. 33)) is clearly visible.

A low heating rate is used $\left(1^{\circ} \mathrm{C} \mathrm{min}^{-1}\right)$ to prevent changes in particle size of the supported metal nanoparticles. ${ }^{13}$ Highresolution TEM images (Fig. 3 and Table 2, Fig. S9 and Table $\mathrm{S} 2 \dagger)$ of various, as-synthesized supported metal nanoparticles
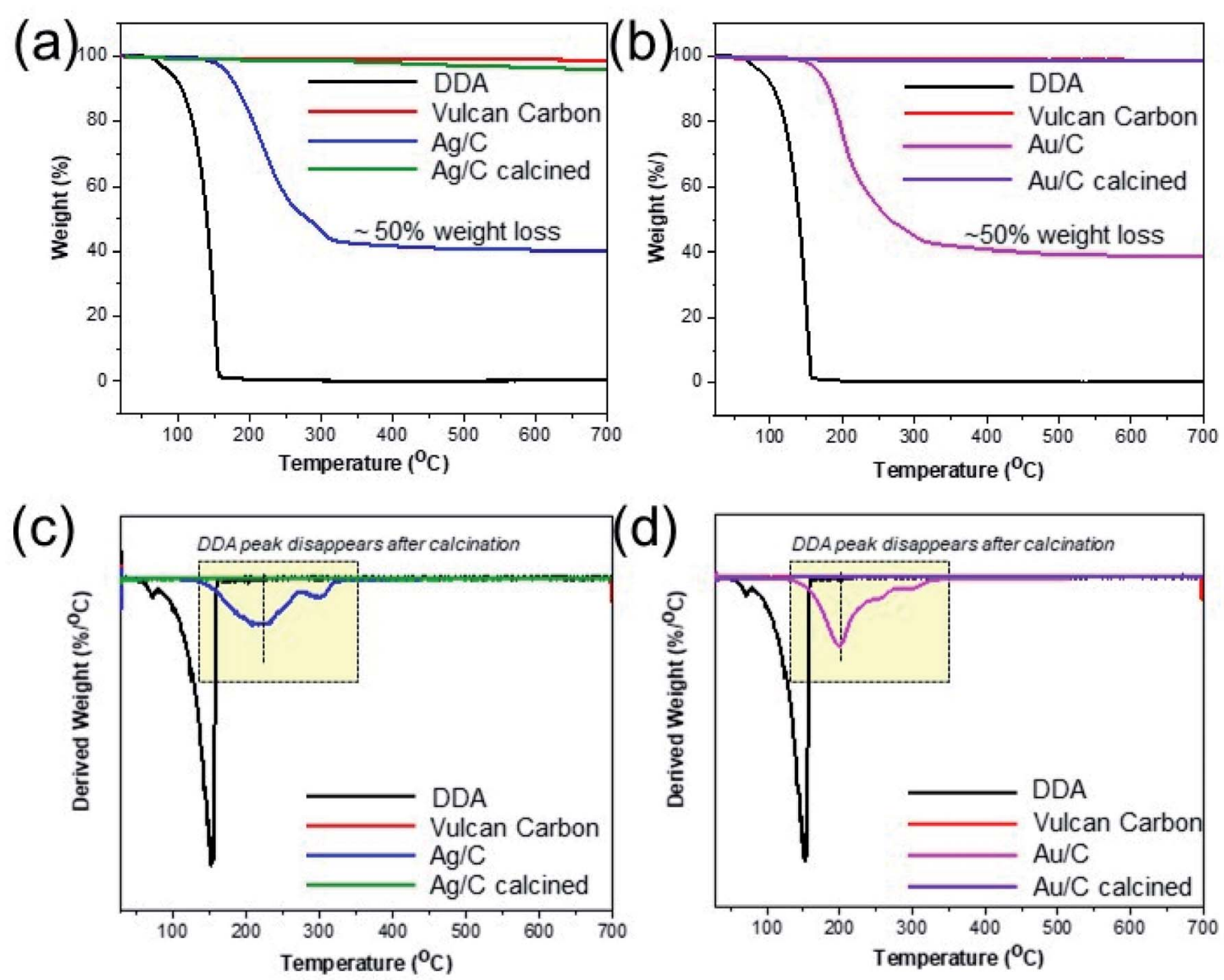

Fig. 2 TGA spectra of $\mathrm{Ag} / \mathrm{C}(\mathrm{a})$ and $\mathrm{Au} / \mathrm{C}$ (b) indicating the required annealing temperature for the removal of DDA surfactant, which is confirmed by DTA analysis ((c) and (d)). 
As synthesized

(a)

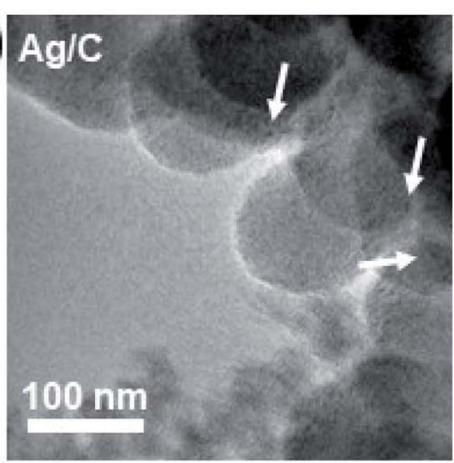

(b)

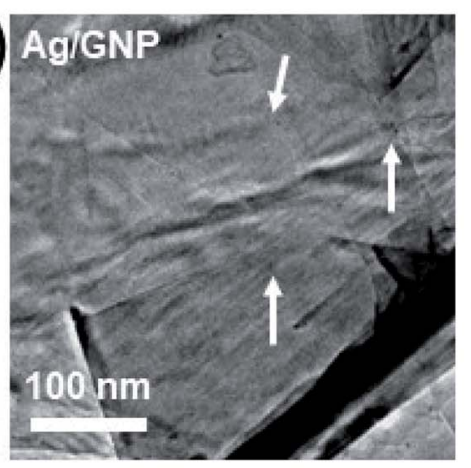

(c)

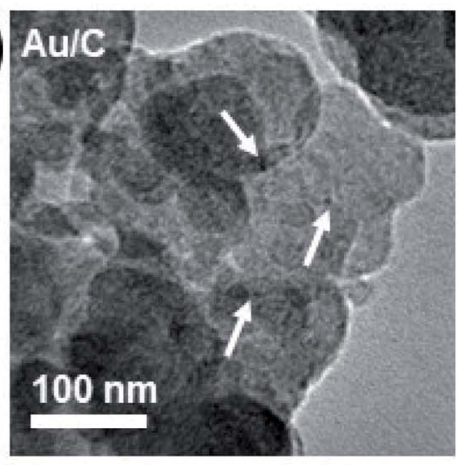

(d)

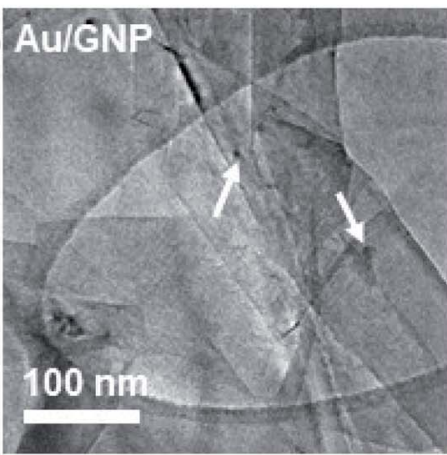

Calcined
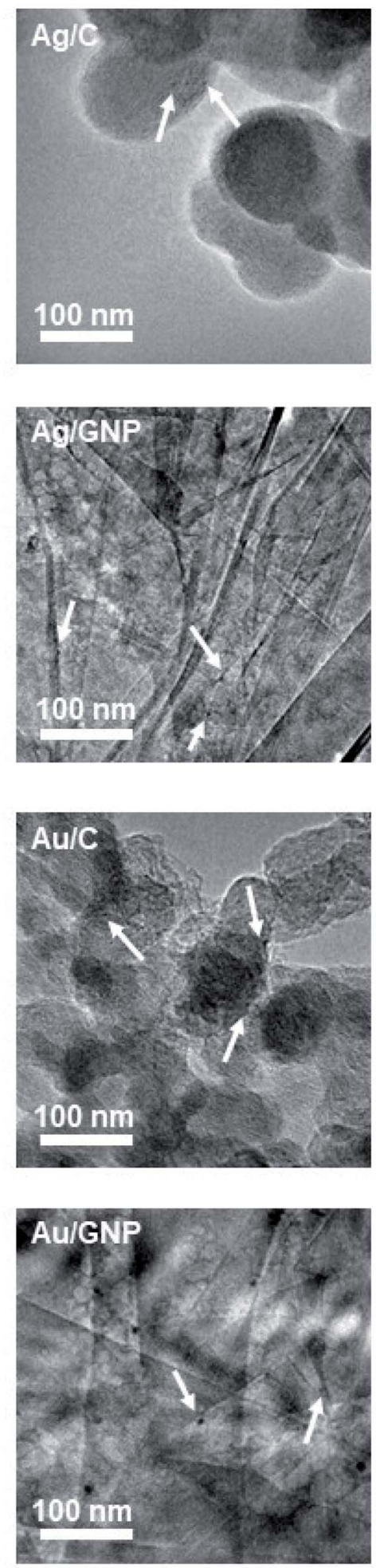

As synthesized

Calcined
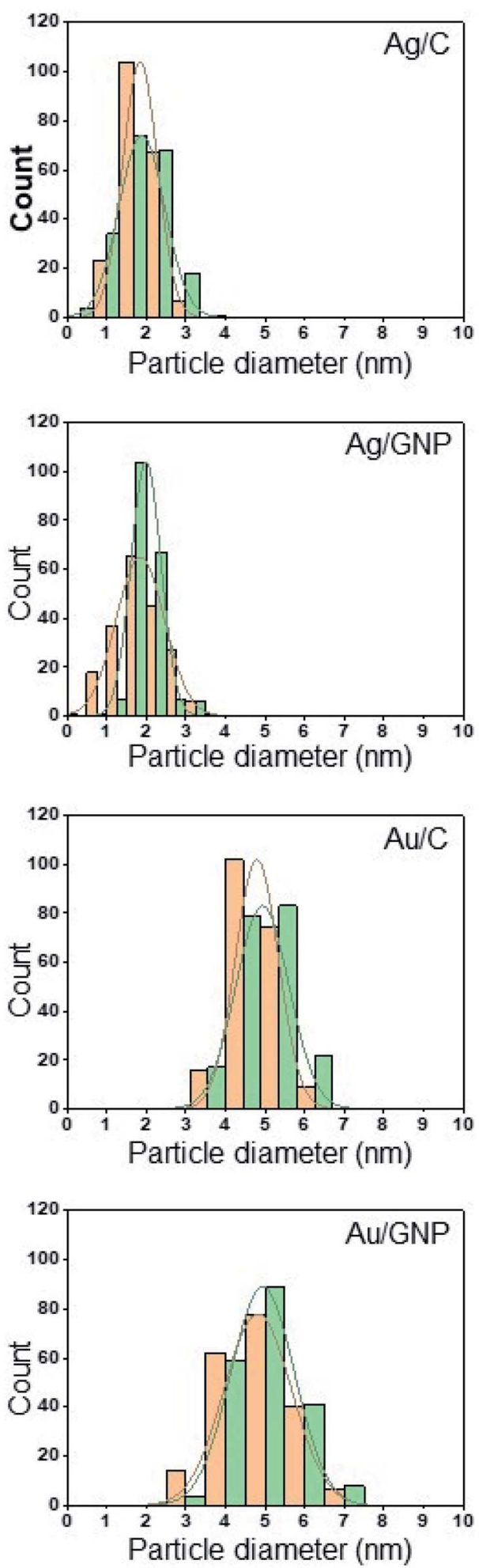

Fig. 3 TEM images of uncalcined and calcined (a) Ag/C, (b) Ag/GNP, (c) Au/C, and (d) Au/GNP, showcasing that their particle size remains intact, post calcination. The histograms are created based on measuring the size of 200 nanoparticles, in each case.

demonstrate that their initial particle size remains intact, post calcination at $350^{\circ} \mathrm{C}$ for $3 \mathrm{~h}$, implying the protective role of DDA on supported metal nanoparticles.
To verify that the surfactant is completely removed from the supported metal nanoparticles post calcination and, hence, the active sites on their surface are not passivated, $\mathrm{Ag} / \mathrm{C}$ is used as 
Table 2 Average size and standard deviation of uncalcined and calcined metal nanoparticles ( $\mathrm{Ag}$ and $\mathrm{Au}$ ) on carbonaceous supports (spraying nozzle i.d. 0.007 inch). Calculation is based on TEM measurements (200 counts)

\begin{tabular}{lll}
$\begin{array}{l}\text { Supported metal } \\
\text { nanoparticles }\end{array}$ & Uncalcined $(\mathrm{nm})$ & Calcined $(\mathrm{nm})$ \\
\hline $\mathrm{Ag} / \mathrm{C}$ & $1.8 \pm 0.4$ & $1.9 \pm 0.6$ \\
$\mathrm{Ag} / \mathrm{GNP}$ & $1.9 \pm 0.6$ & $2.0 \pm 0.4$ \\
$\mathrm{Au} / \mathrm{C}$ & $4.8 \pm 0.6$ & $4.9 \pm 0.7$ \\
$\mathrm{Au} / \mathrm{GNP}$ & $4.8 \pm 0.9$ & $4.9 \pm 0.8$
\end{tabular}

an electrocatalyst for the oxygen reduction reaction in alkaline media $(0.1 \mathrm{M} \mathrm{KOH})$; its specific activity $\left(\sim 0.04 \mathrm{~mA} \mathrm{~cm}^{-2}\right.$ at $0.85 \mathrm{~V} v s$. NHE, $\sim 8 \mathrm{~nm}$ Ag nanoparticle) is in accordance with values reported earlier in the literature (Fig. S14 $\dagger$ ). ${ }^{35-38}$ As expected, ORR activity is reduced as the particle size decreases, due to the increased number of corner sites present on $\mathrm{Ag}$ nanoparticles. ${ }^{36,37}$ On the contrary, uncalcined $\mathrm{Ag} / \mathrm{C}$ does not exhibit any activity, demonstrating that the DDA blocks the access of reactants to the surface active sites.

Apart from thermal annealing, an alternative technique has been used for the removal of the DDA surfactant, namely nonthermal oxygen plasma. We have recently demonstrated that this technique can be successfully utilized for the removal of thiphenylphosphine ligand from the surface of Au/TS-1 to increase the number of available active sites on its surface without altering the size of $\mathrm{Au}$ nanoparticles. ${ }^{39}$ However, the oxygen plasma treatment is not efficient for the removal of DDA: even after subjecting the samples to oxygen plasma irradiation three consecutive times (20 min duration each) at room temperature, DTA analysis of the plasma-treated samples shows the presence of DDA surfactant (Fig. S12†).

\section{Conclusions}

In summary, we demonstrated the effectiveness of electrospraying in preparing single metal nanoparticles with narrow distribution. The diameter of the spraying nozzle plays an important role in the particle size, as the larger the nozzle diameter, the larger the produced particle size. This is very useful for materials chemistry, since it allows the production of nanoparticles with a desired size, tailored to the application. ${ }^{39}$ The effectiveness of surfactant removal is examined via thermal annealing and oxygen plasma irradiation, with thermal annealing in air being the most successful treatment leading to the complete removal of DDA as confirmed by TGA-DTA, FTIR, and XPS measurements. A low annealing rate, in conjunction with an amine stabilizing agent adsorbed onto the supported metal nanoparticles, is a beneficial combination, as it prevents the alteration of their particle size during calcination, while the amine is completely removed by the end of the heating process.

This method to synthesize and incorporate metal nanoparticles of controlled size onto supports, as well as remove a widely used surfactant (DDA) without change in particle size, could be generalized to produce a range of metal nanoparticles from the liquid phase. However, the optimal choice of surfactant acting as a reductive and stabilizing agent is case dependent: even though DDA can successfully reduce $\mathrm{Au}$ and $\mathrm{Ag}$ at room temperature, this is not the case for Pt or Pd nanoparticles, where a stronger reducing agent (such as sodium borohydride) should be added to the DDA solution.

\section{Conflicts of interest}

There are no conflicts of interest to declare.

\section{Acknowledgements}

The Zentrum für Elektronmikroskopie at Tu Berlin (ZELMI) is gratefully acknowledged for support in the microscopic analysis. The authors gratefully acknowledge support from an EPSRC "Frontier Engineering" Grant (EP/K038656/1) and an EPSRC "Frontier Engineering: Progression" Grant (EP/S03305X/ 1). The authors also acknowledge SABIC for financial support.

\section{References}

1 T. Ishida, T. Murayama, A. Taketoshi and M. Haruta, Chem. Rev., 2020, 120, 464-525.

2 M. K. Samantaray, V. D'Elia, E. Pump, L. Falivene, M. Harb, S. Ould Chikh, L. Cavallo and J.-M. Basset, Chem. Rev., 2020, 120, 734-813.

3 Y. Du, H. Sheng, D. Astruc and M. Zhu, Chem. Rev., 2020, 120, 526-622.

4 P. G. Jamkhande, N. W. Ghule, A. H. Bamer and M. G. Kalaskar, J. Drug Delivery Sci. Technol., 2019, 53, 101174.

5 K. Saha, S. S. Agasti, C. Kim, X. Li and V. M. Rotello, Chem. Rev., 2012, 112, 2739-2779.

6 P. Trogadas and M.-O. Coppens, Chem. Soc. Rev., 2020, 49, 3107-3141.

7 P. Trogadas, T. F. Fuller and P. Strasser, Carbon, 2014, 75, 542.

8 P. Trogadas, V. Ramani, P. Strasser, T. F. Fuller and M.-O. Coppens, Angew. Chem., Int. Ed., 2016, 55, 122-148.

9 J. Quinson, S. Neumann, T. Wannmacher, L. Kacenauskaite, M. Inaba, J. Bucher, F. Bizzotto, S. B. Simonsen, L. TheilKuhn, D. Bujak, A. Zana, M. Arenz and S. Kunz, Angew. Chem., Int. Ed., 2018, 57, 12338-12341.

10 A. Heuer-Jungemann, N. Feliu, I. Bakaimi, M. Hamaly, A. Alkilany, I. Chakraborty, A. Masood, M. F. Casula, A. Kostopoulou, E. Oh, K. Susumu, M. H. Stewart, I. L. Medintz, E. Stratakis, W. J. Parak and A. G. Kanaras, Chem. Rev., 2019, 119, 4819-4880.

11 Z. Niu and Y. Li, Chem. Mater., 2014, 26, 72-83.

12 N. Kapil, F. Cardinale, T. Weissenberger, P. Trogadas, T. A. Nijhuis, M. M. Nigra and M.-O. Coppens, Chem. Commun., 2021, 57, 10775-10778.

13 D. Li, C. Wang, D. Tripkovic, S. Sun, N. M. Markovic and V. R. Stamenkovic, ACS Catal., 2012, 2, 1358-1362.

14 H. Tao, T. Wu, M. Aldeghi, T. C. Wu, A. Aspuru-Guzik and E. Kumacheva, Nat. Rev. Mater., 2021, 6, 701-716. 
15 P. F. M. de Oliveira, R. M. Torresi, F. Emmerling and P. H. C. Camargo, J. Mater. Chem. A, 2020, 8, 16114-16141.

16 J. Huang, L. Lin, D. Sun, H. Chen, D. Yang and Q. Li, Chem. Soc. Rev., 2015, 44, 6330-6374.

17 A. Jaworek, J. Mater. Sci., 2007, 42, 266-297.

18 A. Quintanilla, M. Valvo, U. Lafont, E. M. Kelder, M. T. Kreutzer and F. Kapteijn, Chem. Mater., 2010, 22, 1656-1663.

19 Y. Chen and X. Wang, Mater. Lett., 2008, 62, 2215-2218.

20 J. A. Lopez-Sanchez, N. Dimitratos, C. Hammond, G. L. Brett, L. Kesavan, S. White, P. Miedziak, R. Tiruvalam, R. L. Jenkins, A. F. Carley, D. Knight, C. J. Kiely and G. J. Hutchings, Nat. Chem., 2011, 3, 551-556.

21 K. Jia, P. Wang, L. Yuan, X. Zhou, W. Chen and X. Liu, J. Mater. Chem. C, 2015, 3, 3522-3529.

22 S. Yu, Y. Yin, X. Zhou, L. Dong and J. Liu, Environ. Sci.: Nano, 2016, 3, 883-893.

23 A. Zuber, M. Purdey, E. Schartner, C. Forbes, B. van der Hoek, D. Giles, A. Abell, T. Monro and H. EbendorffHeidepriem, Sens. Actuators, B, 2016, 227, 117-127.

24 V. Amendola, R. Pilot, M. Frasconi, O. M. Maragò and M. A. Iatì, J. Phys.: Condens. Matter, 2017, 29, 203002.

25 S. Peng, J. M. McMahon, G. C. Schatz, S. K. Gray and Y. Sun, Proc. Natl. Acad. Sci., 2010, 107, 14530-14534.

26 E. J. Guidelli, A. P. Ramos, M. E. D. Zaniquelli and O. Baffa, Spectrochim. Acta, Part A, 2011, 82, 140-145.

27 Q. Zhang and H. Wang, ACS Catal., 2014, 4, 4027-4033.
28 A. Alehosseini, B. Ghorani, M. Sarabi-Jamab and N. Tucker, Crit. Rev. Food Sci. Nutr., 2018, 58, 2346-2363.

29 N. Arya, S. Chakraborty, N. Dube and D. S. Katti, J. Biomed. Mater. Res., Part B, 2009, 88, 17-31.

30 X.-X. Wang, X.-J. Ju, S.-X. Sun, R. Xie, W. Wang, Z. Liu and L.-Y. Chu, RSC Adv., 2015, 5, 34243-34250.

31 P. Chen, H. Li, S. Song, X. Weng, D. He and Y. Zhao, Results Phys., 2017, 7, 2281-2288.

32 T. T. Nguyen, P. Bandyopadhyay, X. Li, N. H. Kim and J. H. Lee, J. Membr. Sci., 2017, 540, 108-119.

33 M. C. F. Soares, M. M. Viana, Z. L. Schaefer, V. S. Gangoli, Y. Cheng, V. Caliman, M. S. Wong and G. G. Silva, Carbon, 2014, 72, 287-295.

34 M. Aslam, E. A. Schultz, T. Sun, T. Meade and V. P. Dravid, Cryst. Growth Des., 2007, 7, 471-475.

35 M. S. Ahmed, D.-W. Lee and Y.-B. Kim, J. Electrochem. Soc., 2016, 163, F1169-F1176.

36 S. M. Alia, K. Duong, T. Liu, K. Jensen and Y. Yan, ChemSusChem, 2012, 5, 1619-1624.

37 H. Erikson, A. Sarapuu and K. Tammeveski, ChemElectroChem, 2019, 6, 73-86.

38 T. VanCleve, E. Gibara and S. Linic, ChemCatChem, 2016, 8, 256-261.

39 N. Kapil, T. Weissenberger, F. Cardinale, P. Trogadas, T. A. Nijhuis, M. M. Nigra and M.-O. Coppens, Angew. Chem., Int. Ed., 2021, 60, 18185-18193. 